\title{
Two-Dimensional Polycrystalline ZnO Hierarchical Structures as Single-atom Catalyst Supports
}

\author{
Yafeng $\mathrm{Cai}^{1,2}$, Jia $\mathrm{Xu}^{1}$, Yun Guo ${ }^{2}$ and Jingyue Liu ${ }^{1}$ \\ 1. Department of Physics, Arizona State University, Tempe, USA. \\ 2. Key Laboratory for Advanced Materials and Research Institute of Industrial Catalysis, East China \\ University of Science and Technology, Shanghai, China.
}

Free standing two-dimensional (2D) materials with confined thicknesses are of interest not only for their unique physicochemical properties but also their simplicity for fundamental research [1]. Zinc oxide, one of the most widely studied metal oxide semiconductors, has attracted increasing interest because of its potential applications in electronics, energy conversion/storage, catalysis, sensing, etc [2]. We have previously investigated the catalytic behavior of $\mathrm{ZnO}$ nanowire supported $\mathrm{Pd}_{1}$ single-atom catalysts (SACs) for various catalytic reactions [3]. The total surface area of these single crystal $\mathrm{ZnO}$ nanowires is, however, limited to about $20 \mathrm{~m}^{2} / \mathrm{g}$ and it is difficult to load high levels of single metal atoms onto these single crystal nanowires. We report here the synthesis of high surface area and polycrystalline 2D $\mathrm{ZnO}$ hierarchical nanostructures as a catalyst support platform to anchor single metal atoms. Similar to the case of developing 2 $\mathrm{D} \mathrm{Co}_{3} \mathrm{O}_{4}$ for anchoring noble metal atoms [4], our strategy is to fabricate high surface area 2D ZnO nanosheets with many defect sites (grain boundaries, surface steps, vacancies, etc.) to anchor metal atoms or clusters. Recent experiments have demonstrated the potential of SACs for broad applications in important chemical transformations [5]. But stabilizing high loading levels of single metal atoms onto appropriate support materials, especially during catalytic reactions at elevated temperatures, still poses a grand challenge.

The 2D $\mathrm{ZnO}$ was synthesized by a modified hydrothermal method. Briefly, a selected amount of $\mathrm{Zn}\left(\mathrm{NO}_{3}\right)_{2} \cdot 6 \mathrm{H}_{2} \mathrm{O}$ was dissolved in deionized water to make a clear solution and then sodium citrate and hexamethylenetetramine (HMTA) were added. The solution was vigorously stirred for $1 \mathrm{~h}$ in air and the resultant transparent liquid was heated at $95^{\circ} \mathrm{C}$ for $3 \mathrm{~h}$ in a Teflon-lined autoclave. After cooling down the autoclave to room temperature, $2 \mathrm{D} \mathrm{ZnO}$ precipitates were collected, thoroughly washed, and dried at $60^{\circ} \mathrm{C}$ for $12 \mathrm{~h}$. Field-emission SEM and aberration-corrected STEM were used to examine the morphology and atomic structure of the as-synthesized 2D $\mathrm{ZnO}$ materials.

Figure 1a shows a typical SEM image of the as-synthesized 2D ZnO displaying a flowerlike morphology. Statistical analyses showed that the sizes of the $\mathrm{ZnO}$ flowers range from $0.5 \mu \mathrm{m}$ to $3 \mu \mathrm{m}$. Figure 1b, a bright-field STEM image, shows that the ZnO flowers consist of hierarchically structured $2 \mathrm{D} \mathrm{ZnO}$ nanolayers with thicknesses ranging from $\sim 1 \mathrm{~nm}$ to $\sim 5 \mathrm{~nm}$. Atomic resolution HAADF-STEM images (Figure 1c and 1d) show that the 2D ZnO nanolayers are composed of many $\mathrm{ZnO}$ crystallites with an average size of $\sim 4 \mathrm{~nm}$. The presence of many grain boundaries and abundant surface defects such as atomic steps and vacancies provides desired sites for anchoring metal atoms. Detailed analyses of many such atomic resolution images showed that the exposed surfaces of the 2D ZnO nanolayers are predominantly $\mathrm{ZnO}\{10-10\}$ and $\{11-20\}$ surfaces. Rapid calcination or reduction treatment can further modify the grain boundary structures without compromising the integrity of the $2 \mathrm{D}$ nanolayers. Noble metal atoms will be dispersed onto these high surface area polycrystalline $2 \mathrm{D} \mathrm{ZnO}$ nanolayers and their catalytic performances for selected catalytic reactions will be discussed [6]. 


\section{References:}

[1] C Tan et al, Chem Rev 117 (2017), p.6225.

[2] Z L Wang, Mater Today 7 (2004), p.26.

[3] J Xu et al, Chinese J Catal 38 (2017), p.1549.

[4] J Liu, Y F Cai and Yun Guo, Microsc Microanal 23 (Suppl 1) (2017), p.1868.

[5] J Liu, ACS Catal 7 (2017), p.34.

[6] This work was supported by NSF under CHE-1465057. The authors acknowledge the use of facilities within the John M. Cowley Center for High Resolution Electron Microscopy at Arizona State University. Yafeng Cai gratefully acknowledges financial support from the China Scholarship Council.
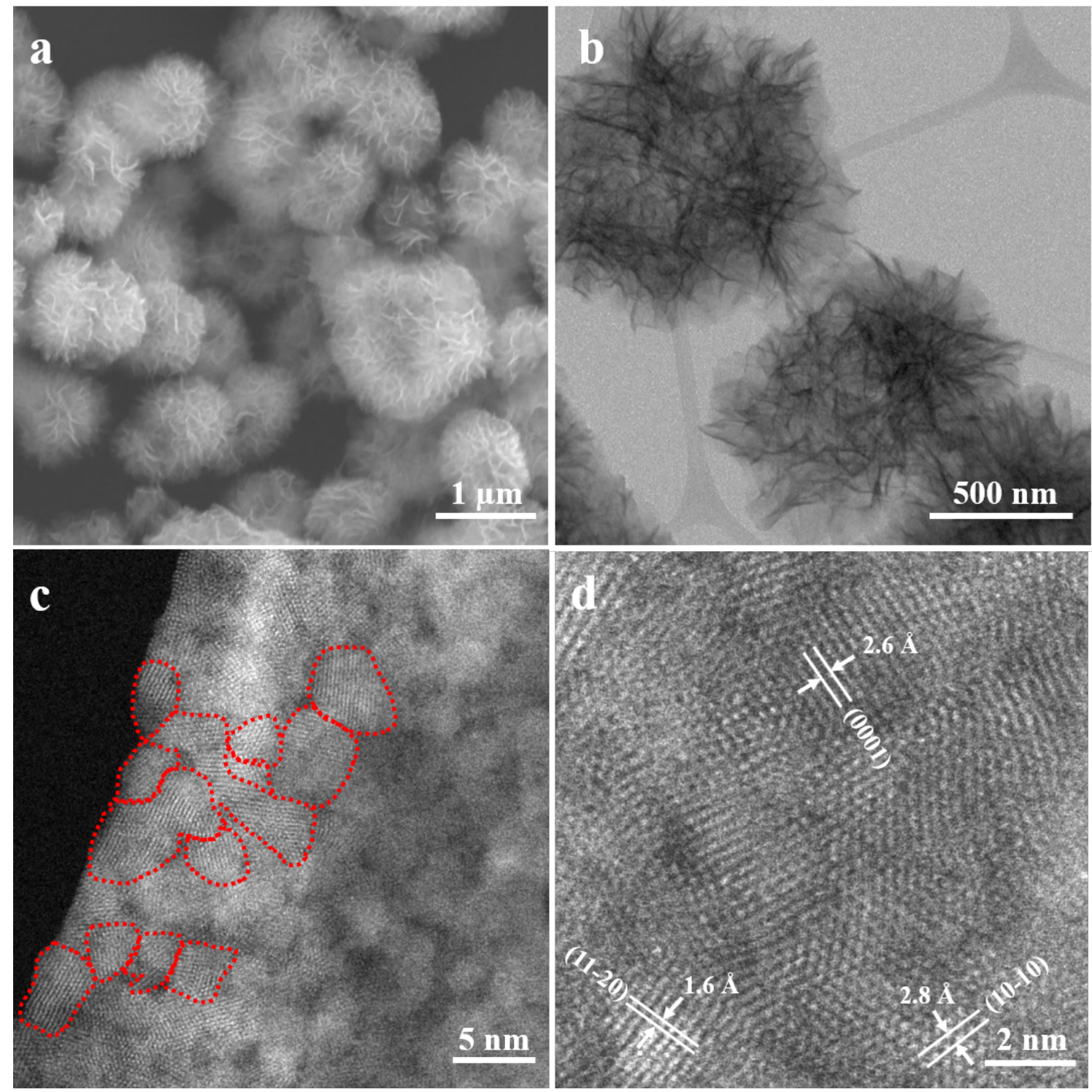

Figure 1. SEM image of the as-synthesized 2D ZnO flowerlike nanostructures (a), a typical bight-field STEM image of individual 2D ZnO flowers, revealing ultrathin $\mathrm{ZnO}$ nanolayers (b), representative high resolution HAADF-STEM images showing the general grain morphology and sizes (c), and the grain boundary orientations and structures (d). 